\title{
Elaboração de um e-book sobre exercícios domiciliares para fadiga em mulheres no climatério
}

\author{
Preparation of an e-book on home exercise for fatigue in weather women \\ Preparación de un e-book sobre ejercicio en casa para la fatiga en el clima mujer
}

Recebido: 06/07/2021 | Revisado: 12/07/2021 | Aceito: 16/07/2021 | Publicado: 21/07/2021

Tatiane Carvalho de Sousa

ORCID: https://orcid.org/0000-0002-5595-2362 Centro Universitário Santo Agostinho, Brasil E-mail: tatiane.carv.sousa@gmail.com

Yarla Katarina Teixeira Morais

ORCID: https://orcid.org/0000-0003-2598-4213 Centro Universitário Santo Agostinho, Brasil E-mail: yarlamorais@hotmail.com

Patrícia Lima Ventura

ORCID: https://orcid.org/0000-0002-8920-2877 Centro Universitário Santo Agostinho, Brasil E-mail: vlpatricia@hotmail.com

\begin{abstract}
Resumo
Objetivo: Elaborar um e-book sobre exercícios domiciliares para a fadiga em mulheres no climatério no formato digital. Metodologia: Trata-se de um estudo transversal-observacional, prospectivo, com abordagem quantitativa. No qual o processo de validação foi composto por 7 especialistas na área temática do e-book utilizando a teoria de Pasquali. Foi considerado o Índice de Validade do Conteúdo de, maior ou igual a 0,78\% para se garantir a validação do material. O público alvo foi composto por 27 mulheres no climatério, para a escolha da amostra seguimos a recomendação proposta por Fehring. Na análise dos dados julgados pelo público-alvo, foram considerados validados os itens com nível de concordância mínimo de $75 \%$ nas respostas positivas. Resultados: Os itens abordados no instrumento de validação do e-book pelo especialistas foram divididos em 2 blocos: estruturas e apresentação, e relevância. Apenas 2 item obteve o índice de concordância $<0,78 \%$, todos os outros itens foram considerados adequado com índice de concordância $>0,78 \%$. De maneira geral, as respostas do público-alvo foram concordantes com relação a organização, estilo da escrita, aparência e motivação. Sendo todos os itens, obteve índice de concordância maior de 75\%. Conclusão: Com este estudo foi possível concluir a importância de elaborar um e-book com base no processo de transmissão de conhecimento e na valorização de experiências entre profissionais de saúde e público-alvo.
\end{abstract}

Palavras-chave: Climatério; Exercício físico; Fadiga; Livros.

\begin{abstract}
Objective: Develop an e-book on home exercises for fatigue in climacteric women in digital format. Methodology: This is a cross-sectional, observational, prospective study with a quantitative approach. In which the validation process was composed of 7 experts in the thematic area of the e-book using Pasquali's theory. The Content Validity Index of greater than or equal to $0.78 \%$ was considered to ensure the validation of the material. The target audience was composed of 27 climacteric women, to choose the sample we followed the recommendation proposed by Fehring. In analyzing the data judged by the target audience, the items with a minimum agreement level of $75 \%$ in the positive responses were considered validated. Results: The items covered in the e-book validation instrument by the experts were divided into 2 blocks: structures and presentation, and relevance. Only 2 item had an agreement rate $<0.78 \%$, all other items were considered adequate with an agreement rate $>0.78 \%$. Overall, the responses from the target audience were in agreement regarding organization, writing style, appearance and motivation. With all items, it obtained an agreement rate greater than $75 \%$. Conclusion: With this study, it was possible to conclude the importance of preparing an e-book based on the process of knowledge transmission and on the valuing of experiences between health professionals and the target audience.
\end{abstract}

Keywords: Climacteric; Exercise; Fatigue; Books.

\section{Resumen}

Objetivo: Elaborar un libro electrónico sobre ejercicios caseros para la fatiga en mujeres climatéricas en formato digital. Metodología: Se trata de un estudio transversal, observacional, prospectivo con enfoque cuantitativo. En el cual el proceso de validación estuvo compuesto por 7 expertos en el área temática del e-book utilizando la teoría de Pasquali. Se consideró el Índice de Validez de Contenido mayor o igual a 0,78\% para asegurar la validación del material. El público objetivo estuvo compuesto por 27 mujeres en menopausia, para elegir la muestra seguimos la recomendación propuesta por Fehring. Al analizar los datos juzgados por el público objetivo, se consideraron validados los ítems con un nivel mínimo de acuerdo del $75 \%$ en las respuestas positivas. Resultados: Los ítems cubiertos en el instrumento de validación 
de libros electrónicos por los expertos se dividieron en 2 bloques: estructura y presentación y relevancia. Solo 2 ítems tuvieron una tasa de acuerdo $<0,78 \%$, todos los demás ítems se consideraron adecuados con una tasa de acuerdo $>0,78 \%$. En general, las respuestas del público objetivo coincidieron en cuanto a organización, estilo de redacción, apariencia y motivación. Con todos los ítems obtuvo una tasa de acuerdo superior al $75 \%$. Conclusión: Con este estudio se pudo concluir la importancia de elaborar un libro electrónico basado en el proceso de transmisión del conocimiento y en la valoración de las experiencias entre los profesionales de la salud y el público objetivo.

Palabras clave: Climaterio; Ejercicio físico; Fatiga; Libros.

\section{Introdução}

O climatério é definido como uma fase biológica da vida e não como um processo patológico, inclui a transição entre os períodos reprodutivo e não reprodutivo da mulher, e geralmente ocorre entre 45 a 65 anos. Durante esse período ocorrem diversos fenômenos fisiológicos, esgotamento dos folículos ovarianos, acompanhado com queda progressiva da secreção de estradiol, culminando com a interrupção definitiva dos ciclos menstruais (Lorenzi, et al., 2005). Com isso surgem os sintomas que variam de mulher pra mulher.

As manifestações mais comuns nesse período são: sensação de fadiga, fogachos, insônia, e variações de humor. Essas alterações ocorrem em razão da queda gradual de hormônios, e levam a maioria das mulheres a vivenciar sinais e sintomas que trazem desconfortos (Miranda \& Ferreira \& Corrente, 2014).

Estima-se que durante o período do climatérico, cerca de 60 a $80 \%$ das mulheres referem algum tipo de sintomatologia (Curta \& Weissheimer, 2020). Sendo a fadiga uma das principais queixas no qual é definida como "uma sensação subjetiva de cansaço físico ao nível de atividade". Ainda, "a fadiga pode se manifestar como dificuldade, incapacidade e redução da capacidade em manter uma atividade. (Zwarts \& Bleijenberg \& Van Engelen, 2008).

As pesquisas mostram que exercícios combinado a movimentos corporais planejados, estruturados e repetitivos, reduz efetivamente os níveis de fadiga e melhora a capacidade funcional (Mock, et al., 2001). Portanto, a prescrição de exercícios se torna de extrema relevância, uma vez que, podem promover uma melhora na qualidade de vida para as mulheres no climatério.

Sendo assim, os exercícios domiciliar é uma alternativa simples, eficaz e segura para pacientes com baixo risco, tendo como objetivo principal exercitar pacientes sobre supervisão indireta que pode ser aplicada por exercícios não supervisionado. (Seixas \& Ricardo \& Ramos, 2016).

Diante desse cenário, as tecnologia usada no dia a dia, permitir construir, disseminar o fluxo de informação tornando sua transmissão satisfatória. Bem como os "E-books ou eletronic books que são publicações digitais ou livros eletrônicos que estão disponíveis na web.", segundo (Mesquita \& Conde, 2008).

Perante o exposto o objetivo dessa pesquisa foi elaborar e validar o $e$-book deixando de estar restringido a espaços físicos concretos como bibliotecas, escolas, universidades, etc. E tornando- se um material na atualidade de fácil acesso para o alívio da fadiga em mulheres no climatério.

\section{Metodologia}

Pesquisa metodológica transversal-observacional (Estrela, 2018), prospectivo, abordagem quantitativa (Pereira, et al., 2018), com foco no desenvolvimento de um e-book a ser utilizado em estratégia educativa para mulheres no período do climatério, promovendo exercícios domiciliares para fadiga. A elaboração do e-book teve início com o levantamento bibliográfico sobre o tema em base de dados: Biblioteca Virtual em Saúde - BVS, PEdro, PubMed e livros, utilizando como descritores as palavras chaves: climatério, exercício, fadiga e e-book, combinados entre si com a utilização dos operadores boleanos: and, or, not.

O material elaborado foi intitulado como: "E-book sobre exercícios domiciliares para fadiga em mulheres no climatério", possui 33 páginas, e aborda os assuntos relacionados ao climatério quanto a sua definição, as fases, fisiologia, manifestações 
mais comuns, importância do acompanhamento médico, conceito de fadiga, exercícios e orientações para alívio dessa sintomatologia, além de conter referências bibliográficas utilizadas.

A população do estudo foi composta por 7 especialistas considerando a Teoria de Pasquali, que recomenda que o número seja entre seis a vinte especialistas para o processo de validação. O público alvo foi composto por 27 mulheres no climatério, para a escolha da quantidade da amostra não probabilística seguimos a recomendação proposta por (Fehring, 1994), que recomenda um número de 25 a 50 sujeitos para a validação de instrumentos.

Para seleção dos profissionais incluídos na amostra, utilizou-se o modelo de Fehring(16) adaptado, no qual os peritos precisariam obter minimamente 5 pontos para que fosse possível a sua participação na análise e na validação do instrumento, avaliando-se possuía tese ou dissertação na área, trabalho publicado na área de interesse, participação em bancas avaliadoras de tese, dissertação ou monografia de especialização que envolvesse a temática, ter experiência como docente em disciplinas da área, atuação pratica com diabéticos, ter orientado tese, dissertação , ou monografia na área de interesse.

A busca dos peritos foi feito através da Plataforma Lattes, no qual foi adotado como critérios de inclusão dos especialistas aqueles que eram especialista na área de Fisioterapia da Saúde da Mulher e que atingiram a pontuação mínima de 5 pontos na escala de Juízes, sendo assim, foram excluídos especialistas que não eram da área de interesse; que não assinaram o termo de consentimento livre e esclarecido.

A carta convite aos profissionais foi enviada por endereço eletrônico, juntamente com o Termo de Consentimento Livre e Esclarecido, o e-book, e um link que dava acesso na plataforma Surveymonkey no qual encontrava o instrumento de avaliação, adaptado de questionário preexistente e dividido em duas etapas. A primeira etapa do instrumento abordava informações acerca da vida profissional e posteriormente itens de avaliação sobre a estrutura e apresentação, assim como a relevância e veracidade do conteúdo; e finalizando, com um espaço destinado às sugestões e propostas.

A seleção das mulheres no climatério foi feita de acordo com os critérios de elegibilidade definidos para a pesquisa, mulheres que estivessem no climatério, que se alto relatassem estar no climatério, que possuíam idade a partir de 45 anos e/ou tinham acesso ao ambiente virtual. Sendo excluídos da pesquisa mulheres analfabetas; que não se identifica estar no período do climatério ou que não assinarão o TCLE. O convite as mulheres no climatério foi enviado através das redes sociais, juntamente com o TCLE que informava as mulheres da justificativa da pesquisa, objetivos, procedimentos metodológicos, riscos e benefícios do estudo. Ao final do TCLE confirmava a participação aquelas que informava o e-mail. O $e$-book elaborado era enviado via email, juntamente com um link que dava o acesso na plataforma Surveymonkey na qual encontra-se o questionário/etapas de Avaliação. O questionário apresenta perguntas pessoais (idade, grau de escolaridade, sobre a pratica de exercícios físicos e se apresenta ou já apresentava sintomas de fadiga) e perguntas relacionadas ao manual acerca dos domínios, organização, estilo da escrita, aparência e motivação do manual, e finalizando, com um espaço destinado para descrever o que acharam do e-book.

A coleta de dados foi realizada no período de maio de 2021 Compuseram a amostra 7 especialistas e 27 mulheres no climatério.

A análise dos dados foi confeccionado na planilha do Microsoft Excel 2013. Em relação à avaliação do e-book de orientações, o instrumento foi organizado em escala tipo Likert, com cinco opções de resposta: 1- insatisfeito, 2- adequado, 3satisfeito, 4- muito satisfeito, NA- Não se aplica. Para validação do e-book pelos especialistas da área, o item e os instrumentos como um todo, devem apresentar Índice de Validade do Conteúdo (IVC) maior ou igual a 0,78\% que mede a proporção dos juízes em concordância sobre determinado aspecto do instrumento. O índice é calculado por meio do somatório de concordância dos itens marcados como "3" e "4" pelos especialistas, dividido pelo total de respostas (Alexandre \& Coluci, 2011). Os itens que não alcançaram o Índice de Concordância adequado foram revisados e comparados com achados da literatura, a fim de alcançar a sua validade. 
$\mathrm{Na}$ análise dos dados julgados pelo público-alvo, foram considerados validados os itens com nível de concordância mínimo de $75 \%$ nas respostas positivas. Os itens com índice de concordância menor que $75 \%$ foram considerados dignos de alteração.

O projeto de pesquisa foi submetido ao Comitê de Ética em Pesquisa do Centro Universitário Santo Agostinho de Teresina (CEP/UNIFSA), de acordo com o preconizado pela Resolução 466/2012, do Conselho Nacional de Saúde, aprovado em 30 de abril de 2021, por meio do parecer $n^{\circ} 4.683 .175$.

\section{Resultados}

\subsection{Consulta a especialistas da área de interesse}

Amostra foi composta por 7 peritos. Quanto a profissão, seis trabalham na área de fisioterapia da saúde da mulher e um em fisioterapia pélvica, sendo todas mulheres, o tempo de formação varia de 7 a 18 anos, atuação na área temática do e-book é de 6 a 17 anos, sendo 5 com titulação de especialistas e 2 em mestrado. A pontuação média dos peritos baseada no modelo de Fehring, adaptado foi de 5,28 pontos, com pontuação mínima de 5 e máxima de 7 pontos.

Em relação ao processo de validação do $e$-book, as opiniões dos peritos $(\mathrm{n}=7)$, foram analisadas de forma quantitativa por meio das respostas dadas ao itens do instrumento de validação que abordou 2 blocos de análise: 1 sobre a estrutura e apresentação, e 2 sobre a relevância do material elaborado.

A Tabela 1 apresenta as respostas dadas pelos peritos, e o índice de concordância de cada item do primeiro bloco (estruturas e apresentação) de validação, que foi composta por 6 itens, na qual o item que avaliava se as informações eram bem estruturada em concordância e ortografia, não alcançou o índice de concordância mínima $0,78 \%$, os demais itens todos alcançaram o índice de concordância de $0,78 \%$.

No segundo bloco (Relevância) de validação composta por 4 itens, verificou a opinião dos peritos em relação a relevância do e-book, sendo o item que avaliava se material estava adequado para ser utilizado pelo público alvo e por profissional da área da saúde da mulher em suas atividades educativas, não alcançou o índice de concordância mínima $0,78 \%$, os demais itens todos alcançaram o índice de concordância de $0,78 \%$.

Tabela 1 - Avalição dos peritos.

\begin{tabular}{|c|c|c|c|c|c|c|c|c|}
\hline \multirow[b]{2}{*}{ ITENS AVALLATNOS } & \multicolumn{8}{|c|}{$N=7$} \\
\hline & & & & 3 & 4 & NA & NR & IVC \\
\hline \multicolumn{9}{|l|}{ Estruturn e apresentaciolio: } \\
\hline \multicolumn{9}{|c|}{ 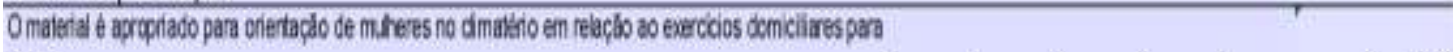 } \\
\hline tajega. & 0 & 1 & 3 & & 3 & 0 & 6 & 0.58 \\
\hline 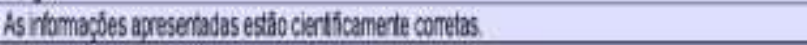 & 0 & 1 & 3 & & 3 & 0 & 6 & 0.88 \\
\hline 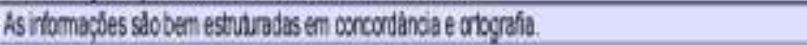 & 0 & 3 & 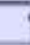 & & 3 & 0 & 4 & 0,57 \\
\hline 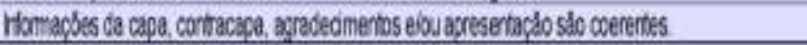 & 0 & 0 & 2 & & 5 & 0 & 7 & 1,00 \\
\hline As ustades sio enpessicase sificertes. & 0 & $\overline{0}$ & 7 & & 4 & 0 & 7 & 1,00 \\
\hline 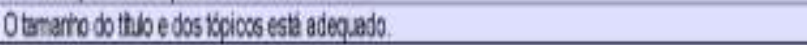 & 0 & 1 & 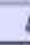 & & 2 & 0 & 6 & 0.86 \\
\hline Total & 0 & 6 & & & 20 & 0 & 36 & 1,00 \\
\hline \multicolumn{9}{|l|}{ Relevinacik } \\
\hline 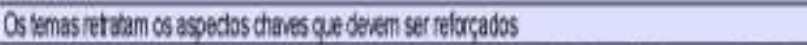 & 0 & 1 & 7 & & 3 & 0 & 6 & 0.85 \\
\hline 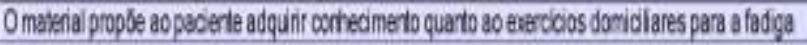 & 0 & 1 & 5 & & 3 & 0 & 6 & $0.8 \%$ \\
\hline 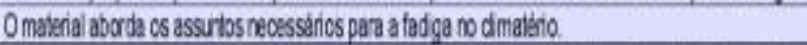 & 0 & 1 & & & 3 & 0 & 6 & 0.88 \\
\hline \multicolumn{9}{|l|}{ 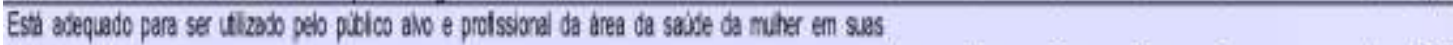 } \\
\hline atvidades edcrabios & 1 & 2 & & & 2 & 0 & 4 & 0.57 \\
\hline Total & 1 & 5 & & & 11 & 0 & 22 & 1,00 \\
\hline
\end{tabular}


A Tabela 1 apresenta as respostas dadas pelos peritos, e o índice de concordância de cada item do primeiro bloco (estruturas e apresentação) de validação, que foi composta por 6 itens. No segundo bloco (Relevância) de validação composta por 4 itens, verificou a opinião dos peritos em relação a relevância do e-book.

Quadro 1 - Sugestões dos peritos.

\begin{tabular}{|c|}
\hline $\begin{array}{l}\text { Estrutura e apresentação: } \\
\text { Estả super didảtico. }\end{array}$ \\
\hline Muita escrita, fica muito sup para ler. \\
\hline Algumas fontes podem ser aumentadas pra facilitar o entendimento das idosas \\
\hline $\begin{array}{l}\text { Falar da atividade fisica especificar mais o acomparhamento com um especialistal } \\
\text { Apenas no final esta escrito que isso năo substitu o acompanhamento de um } \\
\text { profissional. }\end{array}$ \\
\hline Gostei muito das ilustraç̄es e conteưdos. \\
\hline $\begin{array}{l}\text { Sendo impresso, cuidar para năo ficar com fonte muito pequena. Em geral esse público } \\
\text { tem dificuldade para } \\
\text { envergar de perto }\end{array}$ \\
\hline Relevâncla: \\
\hline $\begin{array}{l}\text { Quando a mulher chega nesta fase do climatério, jurto vem uma bagagem de sintomas, } \\
\text { sera que as mulheres iriam consegur realzar a movimentaçăo } \\
\text { adequada com uma cartiha sem a supemstăo de um profissiona? Eu percebo muita } \\
\text { falta de percepçăo corporal para exercicios pélvicos, imagino para uma } \\
\text { mulher q esta sentindo fadiga. Năo quero ser inconveriente, somente a minha colocaçăo. } \\
\text { Talvez utilizaria esses exercicios como preventivo da fadiga no climatério? }\end{array}$ \\
\hline $\begin{array}{l}\text { Incluir mais figuras, pois facilita ainda mais o entendimento a cerca das atvidades } \\
\text { propostas }\end{array}$ \\
\hline $\begin{array}{l}\text { Näo podemos indicar exercicios com resistencia sem conhecer o pacierte. Faltou } \\
\text { oriertaçőes de como começar o exercicio sem resisténcia e evolur conforme } \\
\text { a paciente se sinta segura. Algumas văo começar com um quilo de açúcar por exemplo e } \\
\text { vai ser pesado, mas vai fazer mesmo assim pq ela esta escrito que } \\
\text { precisa e val acabar se lesionando. }\end{array}$ \\
\hline So elogios \\
\hline $\begin{array}{l}\text { Revisāo ortografica pagina } 8 \text {, linguagem estă compreensivel para profissional da área e } \\
\text { para as pacientes?. Fazer revisăo da descriçăo dos movimentos com a } \\
\text { respiração associada principalmente pagina } 20 \text { e verificar os grupos musculares } \\
\text { estimuados, se estảo sendo traba hados agonistas e artagonistas. }\end{array}$ \\
\hline 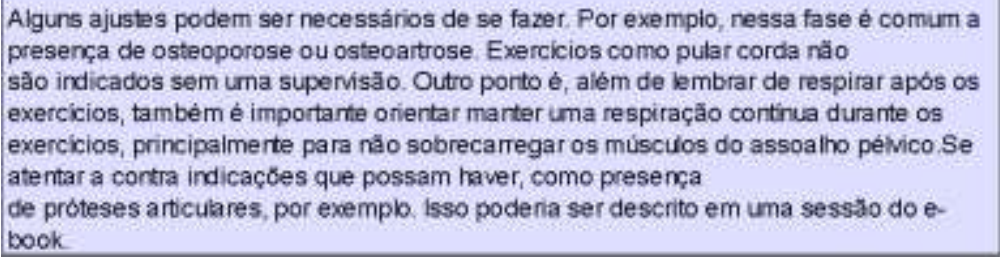 \\
\hline
\end{tabular}

Fonte: Autores (2021).

No Quadro 1 apresenta a síntese das sugestões e comentários realizados pelos peritos em relação ao e-book elaborado. Todas as sugestões apresentadas, relacionadas ou não aos itens que obtiveram Índice de Concordância menor que $0,78 \%$, foram analisadas e avaliadas quanto à sua pertinência para melhor desenvolvimento e aperfeiçoamento do manual.

\subsection{Consulta ao público-alvo}

Amostra do público-alvo foi composta por 27 mulheres no climatério, com idade 45 a 54; na qual 8 tinham como grau de escolaridade ensino fundamental, 13 ensino médio completo e 6 ensino superior. No questionamento sobre a pratica exercícios físicos, 12 relataram praticar e 15 não praticam nenhum exercício. Vale ressaltar que dentre as 27 participantes, 22 já apresentou sintomas de fadiga.

Em relação ao processo de validação do $e$-book, as opiniões das mulheres no climatério $(\mathrm{n}=27)$, foram analisadas de forma quantitativa por meio das respostas dadas ao itens do instrumento de validação que avaliaram a organização, estilo da escrita, aparência e motivação do material elaborado.

A Tabela 2 apresenta as respostas dadas pelas mulheres no climatério, e o índice de concordância de cada item de validação. Na análise dos dados julgados pelo público-alvo, todos os itens foram considerados validados visto que tiveram índice de 
concordância maior que $75 \%$ nas respostas positivas.

Tabela 2 - Avaliação das mulheres em relação a organização, estilo de escrita, aparência e motivação.

\begin{tabular}{|c|c|c|c|}
\hline \multirow{2}{*}{ ITENS AVALLATIVOS } & \multicolumn{3}{|c|}{$N=27$} \\
\hline & 1 & 2 & $10 \%$ \\
\hline \multicolumn{4}{|l|}{ Organizaçào: } \\
\hline A capa chamou sua atençeo? & 27 & 0 & 100 \\
\hline A sequencia do conteudo esti adequedo? & 27 & 0 & 100 \\
\hline A estrima do e-book esta organizada? & 27 & 0 & 100 \\
\hline Total & 81 & 0 & 100 \\
\hline \multicolumn{4}{|c|}{ 1- Sim, 2-Näo; 1 - Indice de Concordância, calcuado pela soma Sim(adequado): Sim x $100 /$ pelo tota Ide respostas. } \\
\hline \multicolumn{4}{|c|}{ Estllo da eserita: } \\
\hline Quarto ao emiondimerto das frasos, obs salo: & 27 & 0 & 100 \\
\hline Conteúdo escrito é & 27 & 0 & 100 \\
\hline Otexto e & 27 & 0 & 100 \\
\hline Total & 81 & & 100 \\
\hline \multirow{2}{*}{\multicolumn{4}{|c|}{ 1-Fácies de entender, 2- Dificeis de entender, $\mathrm{K}$ - Indice de Concordancia, calculado pela soma Fäceis de ertender: Fáceis de entender $\mathrm{x}$}} \\
\hline & & & \\
\hline As ilustraçoes salo: & 27 & 0 & 100 \\
\hline As ilustraçes semem para complamentar o texto? & 27 & 0 & 100 \\
\hline Total & 54 & 0 & 100 \\
\hline \multicolumn{4}{|c|}{$\begin{array}{l}\text { Simples; Complicadas } \\
\text { 1- Sim, 2- Nao; IC- Indice de Concordancia, calculado pela soma Sim(simples) } \operatorname{Sim} \times 100 / \text { pelo total de } \\
\text { respostas. }\end{array}$} \\
\hline \multicolumn{4}{|c|}{ Motivespāo: } \\
\hline $\begin{array}{l}\text { Em sua opsias, quaquer muher no clmatério com } \\
\text { trata? }\end{array}$ & 25 & 2 & 100 \\
\hline Voce se sertiu motivada de ber o e-book ate of fim? & 27 & 0 & 100 \\
\hline \multicolumn{4}{|c|}{$\begin{array}{l}\text { Oe-book aborda os assurtos necessarios para que mukeres no climatério realiznm os oxercicios domicilares } \\
\text { de }\end{array}$} \\
\hline forma adequada? & 27 & 0 & 100 \\
\hline Total & 79 & 0 & 100 \\
\hline
\end{tabular}

Fonte: Autores (2021).

A Tabela 2 apresenta as respostas dadas pelas mulheres no climatério, e o índice de concordância de cada item de validação.

Quadro 2 - Sugestões das mulheres no climatério.

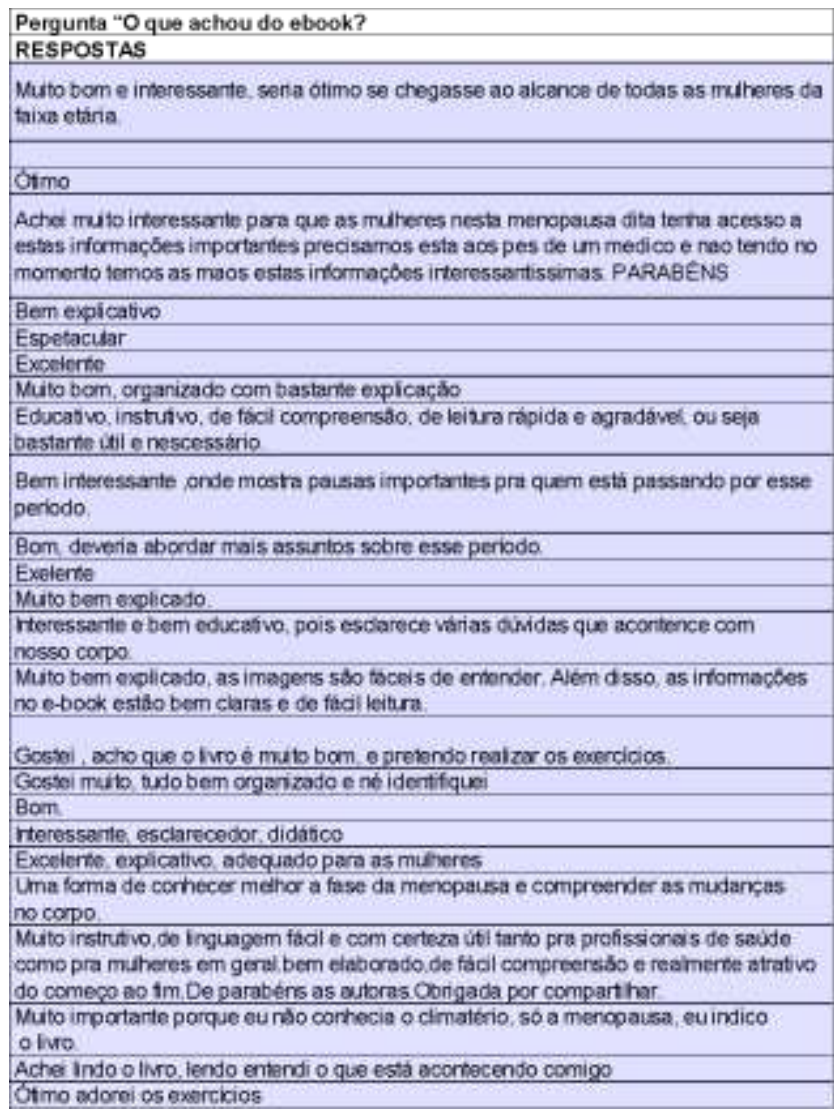

Fonte: Autores (2021). 
No Quadro 2 apresentada a síntese das sugestões e comentários realizados pelas mulheres no climatério em re-lação ao e-book elaborado.

\section{Discussão}

As tecnologias educativas como e-book é uma ferramenta importante para enriquecer a literatura no meio acadêmico e científico, além de trazer benefícios para a sociedade com o modelo atual (e-book), de livre acesso e fácil entendimento. Garantindo maior acessibilidade de conhecimento acerca de sua condição de saúde, promovendo melhor auto cuidado e cooperação juntamente com o profissional de saúde. Dessa forma, a validação do $e$-book torna-se um requisito fundamental para determinar se o instrumento encontra-se adequado para distribuição entre as mulheres no climatério, fisioterapeutas na área da saúde da mulher e demais profissionais. (Vieira, 2015).

De maneira geral, as respostas dos peritos foram concordantes como é possível avaliar na Tabela 1. (Avaliação dos peritos com relação a estrutura, apresentação e relevância). No primeiro bloco (estrutura e apresentação) um item obteve o índice de concordância de $0,57 \%$ ou seja, abaixo do mínimo exigido. Dessa forma as considerações feitas pelos participantes foram acatadas e serão substituídas por informações bem mais estruturadas em concordância e ortografia, tornando uma grafia mais apropriada. Pois é fundamental que a ortografia seja organizada e aprimorada para um texto, caso tenha erros ortográficos e de concordâncias, seu êxito de comunicação é improvável (Santos \& Barrera, 2012).

Nos demais itens relacionados a estrutura e apresentação obteve o índice de concordância maior que $0,78 \%$. Isso mostra que o e-book segundo os especialistas, é apropriado para orientação de mulheres no climatério em relação ao exercícios domiciliares para fadiga, e que o tamanho do título, tópicos estão adequados, além de apresentar conhecimento cientificamente corretos, contendo informações coerentes na capa, contracapa, agradecimentos, apresentação, tendo ilustrações expressivas e suficientes.

Para (Echer, 2005), a construção de materiais de orientação ao cuidado é necessário buscar na literatura especializada, o conhecimento científico existente sobre o assunto. A seguir, é importante transformar a linguagem das informações encontradas na literatura tornando-as acessíveis para toda camada social. É importante procurar, ilustrar as orientações para animar, descontrair e tornar menos pesado, e facilitar o entendimento.

No segundo bloco (relevância) um item obteve o índice de concordância de $0,57 \%$ ou seja, abaixo do mínimo exigido. As considerações feitas quanto a relevância do material de acordo com os especialistas não está adequado, ou seja, não é de fundamental importância para utilizar em atividades educativas. A opinião não foi aceita, pois segundo (Tsuei \& Huang \& Cheng, 2020), os livros digitais é recurso muito utilizado por ser comunicativo, através de ilustrações, animações, e conteúdo, permitindo construir, disseminar o fluxo de informações tornando sua transmissão satisfatória. Sendo assim, o e-book serve para auxiliar nas atividades educativas fora do ambiente formal, de forma eficaz trazendo grandes benefícios. (Farinatti, et al., 2005).

Os demais itens relacionados a relevância obtiveram o índice de concordância maior que $0,78 \%$. Isso mostra que o ebook aborda assuntos necessários que propõe ao paciente adquirir conhecimento. Segundo (Serxner, 2000), um material bem elaborado melhora o conhecimento e a satisfação do paciente, desenvolvendo ações que influenciam no seu padrão de saúde e favorece a tomada de decisão, além de contribuir na redução do uso dos serviços e dos custos com a saúde.

No Quadro 1- (Sugestões dos peritos), no primeiro bloco sobre a estrutura e apresentação do material, todas as sugestões apresentadas foram analisadas, avaliadas e acatadas, quanto à sua pertinência para melhor desenvolvimento e aperfeiçoamento do e-book. No segundo bloco sobre relevância, uma das sugestões dos peritos não foi acatada. Um ponto importante a ser discutido está relacionado a sugestão da não indicação de cartilhas de exercício sem supervisão de um profissional. A realização de exercícios domiciliares tem sido amplamente utilizada segundo (Caglar, et al., 2005) pois contribui para que os indivíduos tenham acesso à exercícios e melhorem a aptidão física. O programa domiciliar apresenta benefícios, sendo imprescindível a orientações para que tenha um resultado satisfatório (Picorelli, et al., 2015). Porem a finalidade da prática de exercício regular em ambiente 
domiciliar é realizar atividade física sob supervisão indireta, ou seja não supervisionados de acordo com (Seixas \& Ricardo \& Ramos, 2016).

Na Tabela 2, de maneira geral, as respostas das mulheres no climatério foram concordantes como é possível avaliar na Tabela 2. (Avaliação das mulheres em relação a organização, estilo da escrita, aparência e motivação). Sendo os itens, obteve o índice de concordância maior de 75\%, tornando possível determinar que o e-book encontra-se adequado para o público alvo. Segundo (Cruz, et al., 2017), acredita-se que a utilização de tais materiais, como estratégias e instrumentos de apoio terapêutico, possa promover conhecimento, e minimizar os efeitos adversos melhorando, assim, a qualidade de vida e o autocuidado.

No Quadro 2- (Sugestões das mulheres no climatério), quando perguntadas “O que achou do ebook?" todas as sugestões apresentadas foram positivas. Considerando o material importante para promoção do conhecimento, com conteúdo motivador aliado à fácil compreensão e leitura agradável, com formato educativo, necessário e explicativo. Além disso, foi mencionada importância de falar mais sobre esse período.

Nesse sentido, de acordo com (Oliveira \& Fernandes \& Sawana, 2008), torna-se relevante a contribuição de tecnologia educativas escrita no contexto da educação em saúde, e o papel desse recurso para promover saúde, prevenindo complicações, desenvolvendo habilidades e favorecendo a autonomia e confiança do paciente. Pois será neste momento em que as mulheres irá trocar informações com maior compreensão do processo que está vivendo, segundo (Mendonça, 2004).

Como limitação deste estudo, pode-se citar a não apreciação do e-book por parte de algumas mulheres no período do climatério, foram encaminhados convites para participação da validação do e-book, sendo feitas várias tentativas de abordagem, porém não houve êxito no retorno.

Tendo-se concluído a construção do $e$-book, o estudo não termina aqui, ressalta-se que o $e$-book passará por atualizações contínuas mediante o progresso científico e tem-se a intenção de levar o material validado para uso nos serviços especializados e de se realizar pesquisas futuras para avaliar a sua eficácia no alcance da implementação das medidas para a redução da fadiga em mulheres no climatério.

\section{Conclusão}

Com este estudo foi possível concluir a importância de elaborar um e-book sobre exercícios domiciliares com base no processo de transmissão de conhecimento e na valorização de experiências entre profissionais de saúde e mulheres no climatério. Portanto, os e-books assumem um papel importante no processo de educação e saúde, visto que facilita a mediação de conteúdos de aprendizagem e funcionam como recurso disponível para que possam realizar exercícios domiciliares.

Diante do observado a necessidade de futuros estudos voltados para a criação de e-books como estratégia de promoção da saúde para as mulheres no climatério.

\section{Referências}

Alexandre, M. \& Coluci, Z. (2011). Validade de conteúdo nos processos de construção e adaptação de instrumentos de medidas. Ciência \& Saúde Coletiva, 16 (7), 3061-3068. https://doi.org/10.1590/S1413-81232011000800006 .

Caglar, A. T. et al. (2005). Effects of home exercises on motor performance in patients with Parkin-son's disease. Sage Journals, 19 (8), 870-877. https://doi.org/10.1191/0269215505cr924oa.

Cruz, F. et al. (2017). Implementação de manuais educativos na consulta de enfermagem: opinião dos pacientes submetidos à quimioterapia antineoplásica. Rev. de Enferm UFPE Online, 11 (5), 1757-62. 10.5205/reuol.11077-98857-1-SM.1105201701

Curta, J. C. \& Weissheimer, A. M. (2020). Percepções e sentimentos sobre as alterações corporais de mulheres climatéricas. Rev. Gaúcha Enferm, 41, 1-9. https://doi.org/10.1590/1983- 1447.2020.20190198.

Echer, I. C. (2005). Elaboração de manuais de orientação para o cuidado em saúde. Revista Latino America, 13 (5), 754-7. https://www.scielo.br/j/rlae/a/6ZJ3s4DtMzZvSJn4JbpD3WB/?format=pdf\&lang=pt.

Estrela, C. (2018). Metodologia Cientifica: Ciência, Ensino, Pesquisa. Editora Artes Médicas.

Farinatti, P. de T. V. et al., (2005). Programa domiciliar de exercícios: efeitos de curto prazo sobre a aptidão física e pressão arterial de indivíduos hipertensos. Rev 
Research, Society and Development, v. 10, n. 9, e25410917933, 2021

(CC BY 4.0) | ISSN 2525-3409 | DOI: http://dx.doi.org/10.33448/rsd-v10i9.17933

Bras Med Esporte, 9 (5), 267-74. 10.1590/S0066-782X2005000600008

Fehring, R. J. (1994). Classification of nursing diagnoses, proceedings of the tenth conference. Philadelphia: Lippincott.

Lorenzi, D. R. S de. et al. (2005). Fatores indicadores da sintomatologia climatérica. Rev. Bras. Ginecol. Obstet., 27 (1), 12-19. https://doi.org/10.1590/S010072032005000100004 .

Mendonça, E. A. P. (2004). Representações médicas e de gênero na promoção da saúde no climatério/menopausa. Ciência Saúde Coletiva, 9 (1), $751-762$. https://doi.org/10.1590/S1413-81232004000100016.

Mesquita, I. C. A. \& Conde, M. G. (2008). A evolução gráfica do livro e o surgimento dos e-books. Sociedade Brasileira de Estudos Interdisciplinares da Comunicação. Teresina: UESPI.

Miranda, J. S., Ferreira, M. L. S. M. \& Corrente, J. E. (2014). Qualidade de vida em mulheres no climatério na atenção Primária. Rev Bras Enferm, 76 (5), 803 9. https://doi.org/10.1590/0034-7167.2014670519.

Mock, V. et al. (2001). Fatigue and Quality of Life Outcomes of Exercise During Cancer Treatment. American Cancer Society, 9 (3), 119- 127. 10.1046 / j.15235394.2001.009003119.x.

Oliveira, M. S., Fernandes, A. F. C. \& Sawana, N. O. (2008). Manual Educativo para o Autocuidado da mulher mastectomizada: Um estudo de validação. Texto Contexto Enferm, 17 (1), 115-23. https://doi.org/10.1590/S0104-07072008000100013.

Pereira, A. S. et al. (2018). Metodologia da pesquisa cientifica. UFSM. https://repositorio.ufsm.br/bitstream/handle/1/15824/Lic_Computacao_MetodologiaPesquisa-Cientifica.pdf?sequence $=1$.

Picorelli, A. M. A. et al., (2015). Adesão de idosas a um programa de exercícios domiciliares pós-treinamento ambulatorial. Fisioter Pesq., 22 (3), 291-308. 10.590/1809-2950/13997522032015.

Santos, M. J. \& Barrera, S. D. (2012). Relação entre conhecimento explícito da ortografia e desempenho ortográfico. Revista Semestral da Associação Brasileira de Psicologia Escolar e Educacional, 16 (2), 257-263. https://doi.org/10.1590/S1413-85572012000200008.

Seixas, M. B., Ricardo, D. R. \& Ramos, P. S. (2016). Reabilitação Domiciliar Com Exercício Não Supervisionado Na DPOC. Rev Bras Med Esporte, 22 (4), 320-25. https://doi.org/10.1590/1517-869220162204150806.

Serxner, S. (2000). How readability of material affects outcomes. Journal of Vascular Nursing, 18 (3), 97-101. https://doi.org/10.1067/mvn.2000.109281.

Tsuei, M., Huang, H. W. \& Cheng, S. F. (2020). Os efeitos de uma estratégia de tutoria por pares na compreensão de leitura de e-books por crianças. South African Journal of Education, 40 (2), 1-12. https://dx.doi.org/10.15700/saje.v40n2a1734.

Vieira, N. N. P. (2015). Validação de manual de condutas para manuseio de cateter totalmente implantado. ENF - Mestrado em Enfermagem (Dissertações), 20119. http://dx.doi.org/10.26512/2015.06.D.18869.

Zwarts, M. J., Bleijenberg, G. \& Van Engelen, B. G. M. (2008). Clinical neurophysiology of fatigue. Science Direct, 119 (1), 2-10. https://doi.org/10.1016/j.clinph.2007.09.126. 